\title{
Results of an Intervention Model in Intestinal Parasitosis in Par- ents Havana 2019-2020
}

\author{
Osvaldo Batista Rojas, MSc* (D) \\ Municipal Unit of Hygiene and Epidemiology, Marianao, Cuba
}

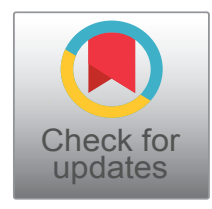

*Corresponding author: Osvaldo Batista Rojas, MSc, Assistant Professor and Researcher, Municipal Unit of Hygiene and Epidemiology, Marianao, Havana, Cuba, Tel: +5356790289; +5372652681

\begin{abstract}
An educational intervention study on intestinal parasitism was carried out in parents of parasitized children of Capitan San Luis de Marianao Elementary School, Havana in the period May 2018-2019, with the purpose of modifying the knowledge in these parents against some hygienic-sanitary aspects in order to reduce the incidence of disease through the application of a program of educational activities. Knowledge was determined through the use of a structured questionnaire. And social activities From a total of 125 parents, a random sample was selected and the questionnaire was administered to 40 parents at the beginning of the study and 6 months after the intervention. Variables such as age, level of education, level of knowledge about the concept, risk factors, routes of transmission, hygienic-sanitary measures and complications of this disease were analyzed. The intervention was effective, allowing satisfactory changes in the level of knowledge of the group studied, with group work constituting an element that allowed enriching and strengthening new methods in order to reduce the incidence of this disease.
\end{abstract}

\section{Keywords}

Parasitism, Parents, Level of knowledge, Intervention, Epidemiology, Environmental risks

\section{Introduction}

Children are likely to acquire parasitic diseases, mainly caused by those parasites whose infectious form penetrates orally. In the transition from breast feeding to preschool age, when they begin their locomotion, their range of action, contact with the environment increases; this increases the risk of contracting parasitic diseases [1].

The patient's age over time has been an important factor when presenting a parasitic infection since the immune system becomes more susceptible to the presence of this infection and therefore tends to control or modulate the infection. This is why the infection may trigger more obvious and serious symptoms in the course of the first years of life. Also, in younger patients, the infection may be more severe due to the immaturity of the system in this pediatric stage. Epidemiological and etiological studies have detected different environmental and individual factors that can influence favoring or hindering of parasitic infection through actions on the parasite or on the host [2-5].

Motivated by the incidence of intestinal parasitic disease associated with unfavorable environmental conditions and the ignorance present in the population and also influenced by not having similar studies carried out in this area beforehand, we determined that this research was vital, with the purpose of modifying the knowledge in these parents in order to reduce the incidence of the disease.

\section{Hypothesis}

If the knowledge of the parents of parasitized children and those already identified in previous studies on risk factors, sanitary hygienic measures, complications, among other aspects related to the disease, will decrease the incidence of intestinal parasitism.

\section{Aims}

Modify knowledge about intestinal parasitism in parents of parasitized children of Capitan San Luis Elementary School, belonging to the Marianao Municipality of Havana in the period of one year.

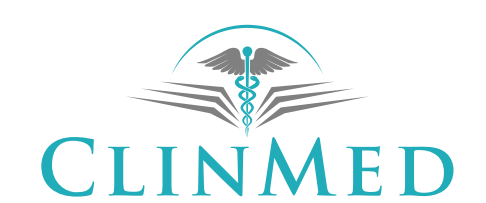

INTERNATIONAL LIBRARY

Citation: Rojas OB (2020) Results of an Intervention Model in Intestinal Parasitosis in Parents Havana 2019-2020. Int J Trop Dis 3:028. doi.org/10.23937/2643-461X/1710028

Accepted: February 18, 2020; Published: February 20, 2020

Copyright: (C) 2020 Rojas OB. This is an open-access article distributed under the terms of the Creative Commons Attribution License, which permits unrestricted use, distribution, and reproduction in any medium, provided the original author and source are credited. 


\section{Material and Methods}

\section{General aspects of the study}

An educational intervention study was conducted with the parents of parasitized children belonging to the Capitan San Luis Elementary School of the Marianao Municipality, Havana in the period from May 20182019 , with the purpose of modifying knowledge about some hygienic-epidemiological aspects referring to this disease through the application of a program of educational activities.

\section{Study universe}

The universe consisted of 195 parents of children and those who gave their consent to participate in the research were included.

The following criteria were taken into account:

\section{Inclusion criteria}

1. Willingness to participate in the intervention.

2. Reside in the health area.

3. Be fit physically and mentally.

4. Be dispensed in the study area.

\section{Exclusion criteria}

1. Not completing the inclusion criteria mentioned above.

\section{Exit Criteria}

1. Leave the investigation.

2. Death or disabling illness during the intervention.

\section{Sample}

A random sample was taken, by simple random method, this being constituted by 40 parents of the group of dispensed children, which represents $56.4 \%$ selected by statistical probability formula.

The following equation was applied to calculate the sample size and its selection so that it is representative:

$$
n=\frac{P(1-\mathrm{P})}{\frac{E^{2}}{Z^{2}}+\frac{P(1-\mathrm{P})}{N}}
$$

Equation for the calculation for qualitative variables and finite population.

\begin{tabular}{|l|l|}
\hline Parameter & It means \\
\hline E & $\begin{array}{l}\text { Permissible difference in proportion. } \\
\text { It is the value of the Confidence Level, in the } \\
\text { normal table. }\end{array}$ \\
\hline P & $\begin{array}{l}\text { Proportion of the population (Percentile of the } \\
\text { normal distribution) }\end{array}$ \\
\hline N & Population size \\
\hline n & Sample size sought. \\
\hline
\end{tabular}

\section{Method}

For the realization of the Educational Intervention, 3 stages were designed:

\section{Diagnostic Stage}

2. Intervention stage itself

\section{Evaluation stage}

\section{Diagnostic stage}

The parents of children who make up the universe were cited and their consent was requested to participate in the intervention, selecting those that were the object of study, who were filled out with the informed consent form (Annex I), and subsequently they were conducted a survey individually (Annex II), qualifying the knowledge before the intervention where the variables of interest for the investigation were taken.

\section{Operationalization of variables:}

Age: Quantitative discretized variable. It was taken in completed years and grouped in a five-year manner. This allowed, together with the classification of the level of schooling, to have an adequate vision of the cognitive sphere of the population under study.

Schooling: Ordinal discrete quantitative variable. Distributed as established by the Ministry of Education.

Level of Knowledge: Qualitative dichotomous variable. Characterized by the items evaluated.

- Adequate.

- Inadequate.

According to evaluation criteria that will be described below:

Information on the knowledge about intestinal parasites was obtained through 8 questions, which had the following evaluation criteria:

- Knowledge about Intestinal Parasites.

- Knowledge about the main risk factors that can influence acquisition of the infection.

- Knowledge of the transmission pathways of Intestinal Parasites. 8 items

- Knowledge on treatment of drinking water.

- Knowledge on the frequency of treating drinking water.

- Knowledge about the correct disposal of solid and liquid residuals.

- Knowledge of sanitary hygienic measures to prevent Intestinal Parasite infection.

- Knowledge about the main complications that a child with this infection could have.

After assessing the knowledge by questions, a general evaluation was carried out, where the total sum of 
all the questions was considered up to a value of 100 points, being evaluated with knowledge of:

- $\quad$ Adequate: If they scored 70 or more points.

- Inadequate: If they scored less than 70 points.

\section{Intervention stage}

The community educational intervention consisted of a program of activities in the form of a 3 month workshop, consisting of 24 hours. This was divide in 8 hours per month, 2 hours per week.

Classes were taught by the author, in a classroom of the same Primary School, Monday and Wednesday from 3 to $5 \mathrm{pm}$.

Participants were divided into two groups methodologically ( $A$ and $B$ ) for the development of the training. The topics were previously updated and reviewed by the author and the tutor. Participatory techniques and educational games were applied.

\section{Evaluation stage}

Information gathering technique: An extensive and thorough literature review of the subject was carried out in the various national and foreign publications available at the National Medical Library of Havana, in addition to the author's experience in other works on the same subject, as well as computerized systems installed, using the information received by Informed and the Internet. The libraries of the Finlay-Albarran Faculty of Medicine were also visited by the Vice Department of Communicable Diseases of the Provincial Center of Hygiene and Epidemiology.

The primary data was collected by the author himself through a face-to-face interview with the parents of the primary school children immersed in the study, to whom a survey (Annex II) was applied before and after the educational intervention.

Information processing techniques: The information obtained before and after the educational intervention was analyzed using the statistical package SPSS, version 11.5.

The information was summarized in double entry tables, using the number and percentage as a summary measure.

The results obtained were presented in containment tables and where there are conditions for their application, the McNemar test was used for a significance of 0.05 and with $95 \%$ reliability ( $p$ less than 0.05 ).

McNemar equation: $((\mathrm{ad})-1)^{2}$

\begin{tabular}{|c|c|c|c|}
\hline \multirow{2}{*}{ Before } & \multicolumn{2}{|c|}{ After } & \multirow{2}{*}{ Total } \\
\cline { 2 - 4 } & Suitable & Inadequate & \\
\hline Suitable & TO & B & $\mathrm{a}+\mathrm{b}$ \\
\hline Inadequate & $\mathrm{C}$ & $\mathrm{D}$ & $\mathrm{c}+\mathrm{d}$ \\
\hline Total & $\mathrm{a}+\mathrm{b}$ & $\mathrm{b}+\mathrm{d}$ & $\mathrm{n}$ \\
\hline
\end{tabular}

Each box expresses the evaluation of the answers being:
a) From adequate to inadequate (+ -)
b) From adequate to adequate $(++)$
c) From inadequate to inadequate (- -)
d) From inadequate to adequate $(-+)$

From the afore mentioned it follows that the boxes a and $d$ are the ones that really measure the effectiveness or not of the intervention.

Analysis technique: We proceeded to analyze all the information obtained through the description and

\section{Parents according to age}

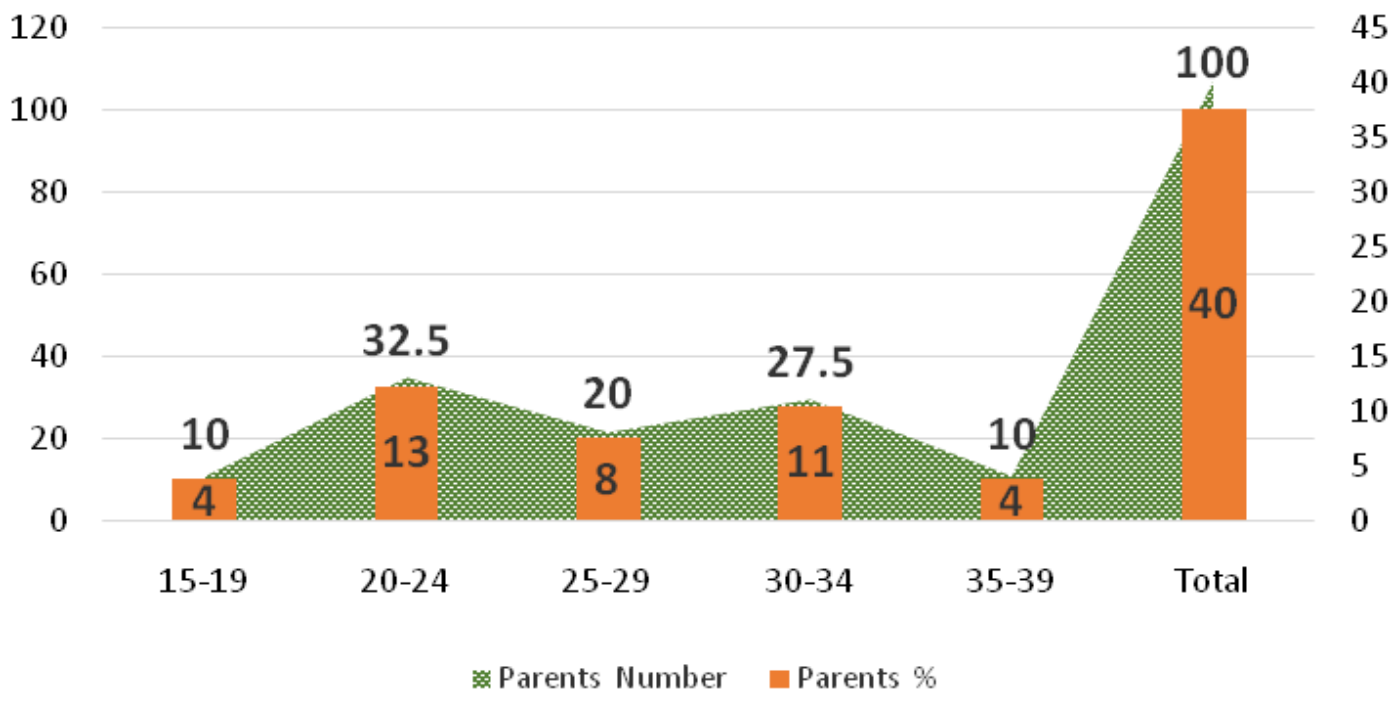

Source: Sociodemographic survey

Figure 1: Distribution of parents according to age. 
discussion of each statistical table and the use of information provided by national and international studies, which allowed us to comply with the proposed objective and reach conclusions. The information was summarized in double entry tables, using the number and percentage as a summary measure.

\section{Ethical aspects}

This study was approved by the Finlay Albarran Medical Sciences Ethical Board.

\section{Results}

In Figure 1, the age distribution of the participating parents is observed, with $32.5 \%$ of the parents belonging to the age group of $20-24$ years $(n=13)$.

Figure 2 shows the distribution of parents by education level, the $70 \%$ of participants had basic secondary level $(n=28)$, followed by $16.6 \%$ with a bachelors ( $n=$ $6)$.
In Table 1, knowledge on the definition of intestinal parasitism is observed, most parents were predominately did not know the definition ( $n=37 ; 92.5 \%$ ). Although after the intervention, there was a $97.5 \%$ modification, that is, only one of the parents still ignored the definition after the intervention.

In Table 2, the risk factors that may influence the onset of the disease were specified, where ignorance prevailed in the parents before the educational intervention, such that $87.5 \%$ considered not treating drinking water, with hand and vegetable washing identified as the most important risk factors.

Through implementation of the program of activities, $95 \%$ of parents were able to acquire better knowledge, allowing the disease to be seen in pediatric ages.

In Table 3, the main routes of transmission were taken into account in order to assess the knowledge that parents had before the intervention, concluding that 34 participants represented by $85 \%$ had a high degree of

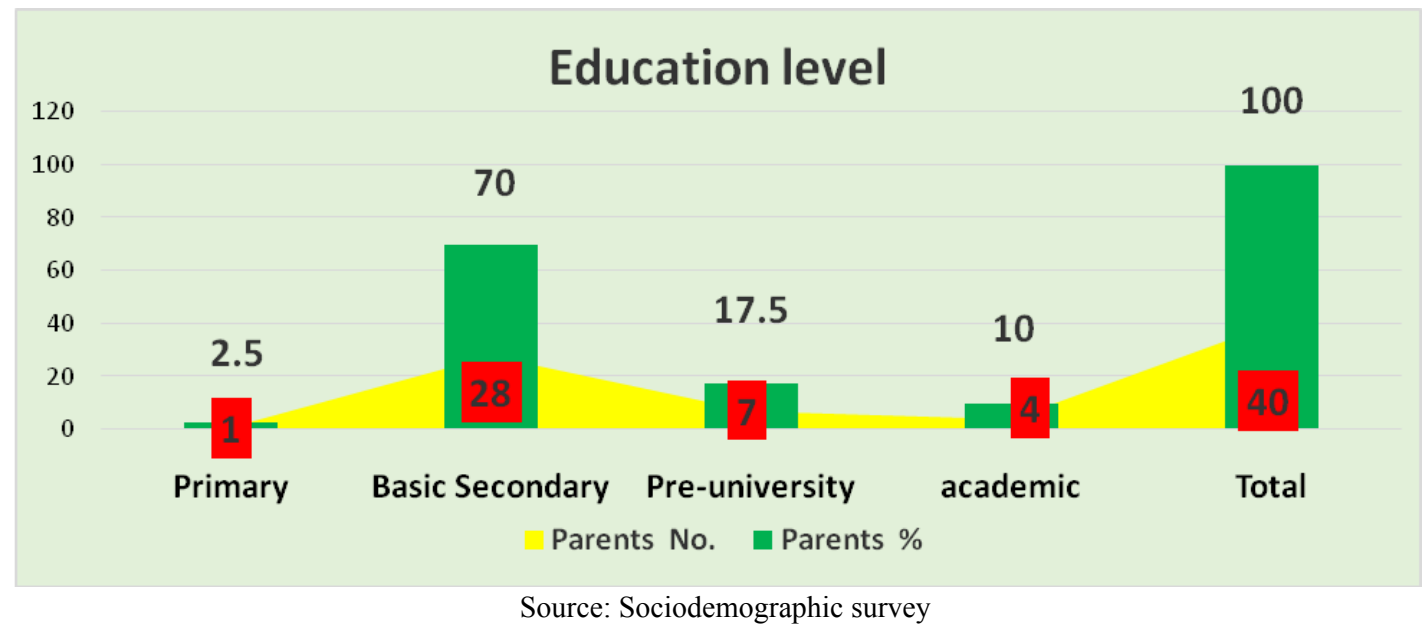

Figure 2: Distribution of parents according to school level.

Table 1: Knowledge about the definition of intestinal parasitism.

\begin{tabular}{|c|c|c|c|c|c|c|}
\hline \multirow[t]{3}{*}{ Before } & \multicolumn{4}{|c|}{ After } & \multirow{2}{*}{\multicolumn{2}{|c|}{ Total }} \\
\hline & \multicolumn{2}{|c|}{ Suitable } & \multicolumn{2}{|c|}{ Inadequate } & & \\
\hline & No. & $\%$ & No. & $\%$ & No. & $\%$ \\
\hline Suitable & 3 & 7.5 & - & - & 3 & 7.5 \\
\hline Inadequate & 36 & 90.0 & 1 & 2.5 & 37 & 92.5 \\
\hline Total & 39 & 97.5 & 1 & 2.5 & 40 & 100 \\
\hline
\end{tabular}

$p<0.05$ Source: Sociodemographic survey.

Table 2: Knowledge of the risk factors of intestinal parasitism.

\begin{tabular}{|c|c|c|c|c|c|c|}
\hline \multirow[t]{3}{*}{ Before } & \multicolumn{4}{|c|}{ After } & \multirow{2}{*}{\multicolumn{2}{|c|}{ Total }} \\
\hline & \multicolumn{2}{|c|}{ Suitable } & \multicolumn{2}{|c|}{ Inadequate } & & \\
\hline & No. & $\%$ & No. & $\%$ & No. & $\%$ \\
\hline Suitable & 5 & 12.5 & - & - & 5 & 12.5 \\
\hline Inadequate & 33 & 82.5 & 2 & 5 & 35 & 87.5 \\
\hline Total & 38 & 95 & 2 & 5 & 40 & 100 \\
\hline
\end{tabular}

$p<0.05$ Source: Sociodemographic survey. 
Table 3: Knowledge of the transmission pathways of intestinal parasites.

\begin{tabular}{|c|c|c|c|c|c|c|}
\hline \multirow[t]{3}{*}{ Before } & \multicolumn{4}{|c|}{ After } & \multirow{2}{*}{\multicolumn{2}{|c|}{ Total }} \\
\hline & \multicolumn{2}{|c|}{ Suitable } & \multicolumn{2}{|c|}{ Inadequate } & & \\
\hline & No. & $\%$ & No. & $\%$ & No. & $\%$ \\
\hline Suitable & 6 & 15 & - & - & 6 & 15 \\
\hline Inadequate & 3. 4 & 85 & - & - & 3. 4 & 85 \\
\hline Total & 40 & 100 & - & - & 40 & 100 \\
\hline
\end{tabular}

$p<0.05$ Source: Sociodemographic survey.

Table 4: Knowledge of the measures to treat drinking water.

\begin{tabular}{|c|c|c|c|c|c|c|}
\hline \multirow[t]{3}{*}{ Before } & \multicolumn{4}{|c|}{ After } & \multirow{2}{*}{\multicolumn{2}{|c|}{ Total }} \\
\hline & \multicolumn{2}{|c|}{ Suitable } & \multicolumn{2}{|c|}{ Inadequate } & & \\
\hline & No. & $\%$ & No. & $\%$ & No. & $\%$ \\
\hline Suitable & 12 & 30 & - & - & 12 & 30 \\
\hline Inadequate & 28 & 70 & - & - & 28 & 70 \\
\hline Total & 40 & 100 & - & - & 40 & 100 \\
\hline
\end{tabular}

$p<0.05$ Source: Sociodemographic survey.

Table 5: Knowledge of the frequency to treat drinking water.

\begin{tabular}{|c|c|c|c|c|c|c|}
\hline \multirow[t]{3}{*}{ Before } & \multicolumn{4}{|c|}{ After } & \multirow{2}{*}{\multicolumn{2}{|c|}{ Total }} \\
\hline & \multicolumn{2}{|c|}{ Suitable } & \multicolumn{2}{|c|}{ Inadequate } & & \\
\hline & No. & $\%$ & No. & $\%$ & No. & $\%$ \\
\hline Suitable & 9 & 22.5 & - & - & 9 & 22.5 \\
\hline Inadequate & 28 & 70 & 3 & 7.5 & 31 & 77.5 \\
\hline Total & 37 & 92.5 & 3 & 7.5 & 40 & 100 \\
\hline
\end{tabular}

$p<0.05$ Source: Sociodemographic survey.

Table 6: Knowledge of the correct disposal of liquid and solid residuals.

\begin{tabular}{|c|c|c|c|c|c|c|}
\hline \multirow[t]{3}{*}{ Before } & \multicolumn{4}{|c|}{ After } & \multirow{2}{*}{\multicolumn{2}{|c|}{ Total }} \\
\hline & \multicolumn{2}{|c|}{ Suitable } & \multicolumn{2}{|c|}{ Inadequate } & & \\
\hline & No. & $\%$ & No. & $\%$ & No. & $\%$ \\
\hline Suitable & 6 & 15 & - & - & 6 & 15 \\
\hline Inadequate & 3. 4 & 85 & - & - & 3. 4 & 85 \\
\hline Total & 40 & 100 & - & - & 40 & 100 \\
\hline
\end{tabular}

$p<0.05$ Source: Sociodemographic survey.

ignorance in the ways in which that intestinal parasites are transmitted. Achieving a satisfactory result after the intervention reaching $100 \%$ knowledge,

In Table 4, the level of knowledge shown of measures to treat drinking water, noting that before the educational intervention $70 \%$ of parents under study were unaware of their importance. This situation was reversed as $100 \%$ of the participants knew what the measures were to treat the water and its purpose from several points of view six months after the intervention, with the aim of improving its quality.

In Table 5, we observe the frequency of drinking water treatment, in which $77.5 \%$ of parents never tried, reversible situation immediately after the educational intervention, which we see in the result that the fre- quency of treating drinking water increased to Always in $92.5 \%$ of the participants.

In Table 6, the improper collection of liquid and solid waste by parents is observed, with $85 \%$ of them not disposing them properly, $62 \%$ of parents mainly use street garbage disposal, 70\% performed their physiological needs in latrines, which do not meet sanitary hygienic requirements. This favors the appearance of vectors, negative factors for the child population which suffers more strongly from intestinal parasitism. After the intervention it was achieved that $100 \%$ of the participants knew the correct form of waste collection and its disposal.

In Table 7, the sanitary hygienic conditions related to intestinal parasite infection as observed; unfavorable 
Table 7: Knowledge of sanitary hygienic measures.

\begin{tabular}{|c|c|c|c|c|c|c|}
\hline \multirow[t]{3}{*}{ Before } & \multicolumn{4}{|c|}{ After } & \multirow{2}{*}{\multicolumn{2}{|c|}{ Total }} \\
\hline & \multicolumn{2}{|c|}{ Suitable } & \multicolumn{2}{|c|}{ Inadequate } & & \\
\hline & No. & $\%$ & No. & $\%$ & No. & $\%$ \\
\hline Suitable & 7 & 17.5 & - & - & 7 & 17.5 \\
\hline Inadequate & 33 & 82.5 & - & - & 33 & 82.5 \\
\hline Total & 40 & 100 & - & - & 40 & 100 \\
\hline
\end{tabular}

$p<0.05$ Source: Sociodemographic survey.

Table 8: Knowledge of the complications of intestinal parasitism.

\begin{tabular}{|c|c|c|c|c|c|c|}
\hline \multirow[t]{3}{*}{ Before } & \multicolumn{4}{|c|}{ After } & \multirow{2}{*}{\multicolumn{2}{|c|}{ Total }} \\
\hline & \multicolumn{2}{|c|}{ Suitable } & \multicolumn{2}{|c|}{ Inadequate } & & \\
\hline & No. & $\%$ & No. & $\%$ & No. & $\%$ \\
\hline Suitable & 3 & 7.5 & - & - & 3 & 7.5 \\
\hline Inadequate & 37 & 92.5 & - & - & 37 & 92.5 \\
\hline Total & 40 & 100 & - & - & 40 & 100 \\
\hline
\end{tabular}

$p<0.05$ Source: Sociodemographic survey.

Table 9: Parental knowledge about intestinal parasitism before and after the educational intervention.

\begin{tabular}{|c|c|c|c|c|c|c|}
\hline \multirow[t]{3}{*}{ Before } & \multicolumn{4}{|l|}{ After } & \multirow{2}{*}{\multicolumn{2}{|c|}{ Total }} \\
\hline & \multirow{2}{*}{$\begin{array}{l}\text { Suitable } \\
\text { No. }\end{array}$} & \multicolumn{3}{|c|}{ Inadequate } & & \\
\hline & & $\%$ & No. & $\%$ & No. & $\%$ \\
\hline Suitable & 8 & 20 & - & - & 8 & 20 \\
\hline Inadequate & 30 & 75 & 2 & 5 & 32 & 80 \\
\hline Total & 38 & 95 & 2 & 5 & 40 & 100 \\
\hline
\end{tabular}

$p<0.05$ Source: Sociodemographic survey.

sanitary hygienic conditions was performed by a total of 33 participants $(82.5 \%)$, values present before applied the educational intervention, being able to assess after using it the level of knowledge achieved by parents rose to $100 \%$.

In Table 8, the ignorance of the complications of parasitic infection present in the parents is observed, which represents $92.5 \%$, some erroneous aspects were identified as abdominal pain with $60 \%$, after the educational intervention we managed to raise the knowledge of parents to $100 \%$ explaining also that there are symptoms that are aggravated and at the same time constitute complications.

Table 9, the evaluation of the knowledge of parents before and after the educational intervention, the aspects evaluated, which were predominantly represented by 32 participants inadequate for $80 \%$.

However, after carrying out the program of activities, it was possible to modify favorably the knowledge of the participants, as can be seen in Figure 1, and raise them to $95 \%$.

The results obtained guarantee the applied intervention as satisfactory, being able to modify the knowledge with statistically significant results for $p$ $<0.05$.

\section{Conclusions}

The intervention carried out through the application of a program of educational and participatory activities was effective, allowing satisfactory changes in the level of knowledge of the group studied, constituting group work an element that allowed enriching and strengthening new methods in order to reduce the incidence of this disease.

\section{Discussion}

At present, the modality of educational intervention has gained great importance, since positive results have been obtained in vulnerable groups in the community [6].

The Educational Intervention has been effective in different health matters in which it has been used, such is the example of the School for hypertensive, diabetic, asthmatic, among others, obtaining satisfactory results, since morbidity and mortality due to other causes have decreased, ensuring that trained patients are able to know their disease better and develop rules of their own behaviors for each of them and that they become facilitators and disseminators of the knowledge acquired, encouraging the masses to incorporate [6-8].

In order to carry out community intervention stud- 
ies it is very important to determine the variables: Age and Level of education.

The age for the contribution of the experiences offered to the group and the school level greatly influences the state of health of the population, because in one way or another the cultural hygienic level of each community decreases or increases, the degree of awareness, reaching or not to know the main problems and factors that affect health and actively participate with health workers in activities with a view to modifying or eliminating these factors [9-11].

Low levels of schooling negatively influence the individual and collective health of the population since health actions are not fully assumed by the low health culture [12].

The evidence of a high intellectual level leads to a low incidence of diseases, this means that as the intellectual level improves, there is a lower risk of suffering from intestinal parasites, given the total sanitary hygienic measures necessary to avoid it, so it is proposed a low incidence in developed countries and its high in underdeveloped countries [13-15].

The results obtained by the author do not coincide with the research published in Mexico in 2007 where the authors conducted a study on 429 children between 6 and 36 months of age and obtained information about the family and their family environment, to relate it to the incidence of intestinal parasitism, when analyzing the schooling of mothers found that the average schooling was 10 years, which corresponds to the first grade of upper secondary education; and that $52.5 \%$ of the mothers had less than average schooling [16].

It can be added that schooling acts in a proportional way since by presenting a better schooling and level of instruction, a greater number of measures appear to avoid the presence of intestinal parasitism.

Some research developed by Ares Maza [17-21], has shown that parents have significant direct or indirect influences on their children from very early ages. All this suggests that the role of the father in family education should be better reviewed due to the different position that he could occupy in certain socio-economic and cultural contexts.

Considering that this constitutes the first step to be able to raise the level of knowledge regarding the disease, making it possible for parents to define it and thus be able to talk about it with a solid foundation based on knowledge.

Similar results were in a study conducted with mothers of children from 0 to 4 years of age at the Corralillo office in the province of Guantanamo in 2007 [22].

The most effective measure in the prevention of any disease is the elimination of all risk factors and predisposing factors to it. Control over all environmental factors must be maintained. All possible disinfection techniques should be used, especially food, objects or animals with which we interact [23].

Water is an important transmission pathway for intestinal parasites and therefore it is important for the population to be aware of treatment options for water that will be used for human consumption, which will constitute a risk factor for the disease as well as a hygienic measure to prevent infection by intestinal parasites.

From the sanitary hygienic point of view, bacteria, excessive mineralization, high levels of protozoan compounds and other organisms are eliminated. From the aesthetic point of view, the correction of smell, taste and turbidity is pursued. From an economic point of view, we are interested in eliminating corrosives [24].

Water is the natural habitat of many pathogenic species, whose abundance and diversity is determined by the availability of dissolved nutrients, water and food such as vegetables that are related here has great epidemiological importance as a vehicle for biological transmission of some parasitic agents, a high incidence of intestinal parasitism was reported in a study carried out last year in patients where the administration of unwashed food and improperly treated or improperly purified water is appreciated [24].

This study coinciding with others that showed that houses with little or no drinking water service and that are not treated, the inhabitants have greater infection by parasites, together with the lack of health education, total ignorance shown by the representatives of children from the most elementary hygienic measures that must be taken into account [25].

As much as the hygiene of food as boiling water for daily consumption is very important and that every person must know and be aware that with these measures a large part of the battle in the eradication of diseases such as parasitism is won intestinal.

\section{References}

1. Golsmith R, Heyreman D (1995) Parasitology and Tropical Medicine. The Modern Manual, 314-322.

2. Rozman Farreras (2000) Section Parasitic infections. In: Internal Medicine. Volume 1, (14 ${ }^{\text {th }}$ edn), Spain.

3. (2008) Pediatrics. Scientists Ernesto de la Torre Montejo and Eduardo José Pelayo González Parada. Editorial Medical Sciences, Havana.

4. Botero D, Restrepo M (1996) Intestinal and genital protozoa. In: Restrepo A, Robledo J, Beyoda, Fundamentals of Medicine: Infectious diseases. ( $5^{\text {th }}$ edn $)$, Medellin Corporation for Biological Research, 541-543.

5. Juan E, Calderín Campbell, Emma E (2008) Parasitic diseases and their consequences for human health. 
6. Alarcón de Noya B, Contreras R, Ruiz R, Hernán A, Bruces $A C$, et al. (1999) Intestinal parasites in communities in the central area of Venezuela. XIV Latin American Congress of Parasitology 27: 41-74.

7. Pérez Armengol C, Ariza Astolfi C, Úbeda Ontiveros JM, Guevara Benítez D, de Rojas Álvarez M, et al. (1997) Epidemiology of childrens's intestinal parasitism in the Guadalquivir valley, Spain. Rev Esp Salud Publica 71: 547-552.

8. Núñez Fernández FA, Cordoví RA (2007) Manual of Basic Techniques for the diagnosis of Intestinal Parasitosis. IPK teaching publications, MINSAP-UNICEF.

9. (1999) Parasitic infection section. $\left(10^{\text {th }}\right.$ edn), Manuel de Merck.

10. https://www.mcgill.ca/parasitology.

11. Ruiz Andrados D (2006) Acute diarrheal diseases. Rev Chilena Salud Rural 10: 15-22.

12. David G (2005) World Wide Virtual Library: Parasitological link related to parasitology, complementally. 10: 15-22.

13. Duke S (2006) Coproparasitological examination in children. Biomedical Rev 15: 30-47.

14. Danner J (2007) Ectoparasites and endoparasites academic link and information about fasciola and other parasic. Books S: 131-138.

15. Gamarra Díaz H, Rivero Castro E (2006) Prevalence of intestinal parasitosis in children. Rev Chilena Epidemiológica 3: 131-148.
16. Jiménez Albarran M (2006) Prevalence of intestinal parasitism in emigrants from different parts of the world. 3: 22-40.

17. Hall L (2006) Molecular and biochemical parasitology. Journal for all Scientists that work with parasites as model for their biochemical and molecular studies 2: 120-29.

18. http://www.aeped.es/protocolos/index.htm.

19. Rashidul Haque (2007) Human intestinal parasites. J Health Popul Nutr 25: 387-391.

20. Catalán J, Ariza C, de Rojas M, Libeda JM, Guevara DC (2008) Epidemiology of childhood intestinal parasitism in the state of Chiapas. Rev Mex Microbiol Clin 7: 265-270.

21. Álvarez Hernández A (1993) Prevalence of intestinal parasitism in supposedly healthy children. Cuban Rev General Comprehensive Medicine 9: 220-222.

22. Duke S (1994) Coproparasitological examination in children. Rev Biomed 14: 30-47.

23. Martínez C (2004) Incidence of E Nana as the first cause of parasitism in children in South. 3: 3-5.

24. Castillo Acosta $M$, Toledo Cúrvelo $G$, Tejeiro Fernández $A$, Rodríguez Hernández P, Martínez Calvo S, et al. (1998) Epidemiology. Editorial People and Education, Havana City, 79-80.

25. Núñez Fernández FA (2001) Giardia Lamblia. In: Lopa Valdés-Dapena M, Suazo L, Medical Microbiology and Parasitology. Volume III, Editorial Medical Sciences, Havana City, 31-38. 


\section{Annex I: Informed Consent.}

\section{(Parents, Guardians and Representatives)}

Intestinal diseases caused by parasites affect about a third of the world's population. Those people who live in developing countries, particularly those of tropical climate, have the tendency to be the most affected, adding the adverse social and economic conditions in which they develop, a very vulnerable group are children, bringing symptoms that sometimes can be serious for infants.

Parent or guardian I give my disposition so that my son is included in the intervention study on knowledge of intestinal parasitism in children belonging to our community.

I know that my son's participation is absolutely voluntary, that he can leave the study without my rights being affected and that he will be able to get medical attention if he needs it in the popular doctor's office.

I consider it very important to carry out this study in our country because parasitosis is a disease that involves a risk for a significant number of people, especially children, and with the participation of my son, I will contribute to the control and prevention of them.

Volunteer Signature

\section{Annex II}

\section{Questionnaire for General Information on Intestinal Parasitism.}

\section{Do you know what Intestinal Parasitism is?}

Have you ever had intestinal parasites or do you know any of your friends who have ever been sick because of this? Then and with all the desire to help you to increase your knowledge about it, we show you a series of questions that you must answer with all sincerity, so that later, the area doctor can help you clarify all your questions. Thank you very much for your cooperation.

a) Educational level:

1- What do you understand by Intestinal Parasitism and what are the types of Parasites you know? Mark the correct answers.

a) __ Disease that plants have.

b) _ Condition that can affect children and men who do not take good care of their health, and occurs within our digestive system (belly or belly), causing damage.

c) __ It never heals.

d) __ It is transmitted from one person to another.

e) __ Parasites are divided into: Protozoa (microscopic, which can not be seen with the naked eye) and helminths (worms). 
f) __ Not all parasites are seen in the stool (poop).

2- Which of these proposals do you think are the ones that favor the appearance of the disease? Select them.

a) _ Greeting other children who have the disease.

b) __ Eat without having washed your hands previously.

c) __ Fruits and vegetables that are eaten without washing them first.

d) __ Drink unboiled water.

e) __ Consume street foods that are not hygienically prepared.

f) __ For walking or playing barefoot.

g) __ Do not keep nails cut and clean.

h) _ Do poop outdoors.

i) __ Keep garbage uncovered.

j) __ Eat meat that has not been sufficiently cooked.

k) _ Bathing in pools.

3- Mark with an $\mathrm{X}$ what you think a child or anyone could feel if he had parasites.

a) _ Pain and swelling of the stomach.

b) __ Diarrhea (sometimes bloody).

c) _ Constipation.

d) __ Itching in the anus (buttocks).

e) __ Presence of earthworms or worms in the poop.

f) __ Decay or fatigue.

g) __ Lack of appetite.

4- From the following statements, you must choose which ones help prevent or prevent the onset of the disease.

a) __ Play with dirt.

b) _ Wash hands thoroughly before handling, eating and after going to the bathroom.

c) __ Drink pure or boiled water.

d) __ Keep nails short and clean.

e) __ Do not poop outdoors.

f) __ Keep the garbage tanks covered.

g) __ Do not walk barefoot.

h) _ Covering kitchen utensils and food with clean tablecloths, to prevent flies from falling on them.

i) __ Cooking enough food, mainly meats.

j) __ Do not buy food or spare parts in street sales that we do not see with hygiene (clean).

k) _ Take water from the jet and do not keep your hands clean.

5- What do you think should be done to any patient with Intestinal Parasitism?

a) _ Maintain the usual diet.

b) __ Go to the Health Center, so that doctors and nurses can cure it.

c) __ Raise the hygiene and cleaning measures in the house, and with the sick child.

d) __ Separate the patient's eating vessels to prevent the disease from being transmitted to the rest of the family members.

e) _ Leave it in the house and give it home remedies without having been attended to 
before the doctor.

Score 30 points in total, ( 6 for each question, 1 for each subsection).

0-10 Poor, 11-20 Average, 21-30 adequate.

Thank you very much for your cooperation.

\section{Survey Applied to Parents, Guardians or Representatives.}

Survey on the behavior of intestinal parasitism in a supposedly healthy infant population.

For your child say:

1. Age

2. Sex

3. Schooling:

Analphabet

Primary-

Technicians

Bachelor-

Academic

4. For which of these symptoms have you assisted the doctor with your child?

Abdominal pain

Anorexy

Diarrhea

Sickness

Vomiting

Urticaria-

Constipation

5. Hygienic habits of the child.

5.1. Are you aware of washing the child's hands before eating?

a). Never:

b). Sometimes:

c). Forever:

5.2. Are you aware of the washing of the child's hands after defecation?

a). Never:

b). Sometimes:

c). Forever:

5.3. Do you allow me to walk barefoot?
a). Usually:
b). Sometimes
c). Never:

5.4. Do you play with dirt?

a). Yes:

b). Sometimes:

c). Never:

\subsection{Nail biting:}

a). Yes:

b). Sometimes:

c). Never:

6. Conscious of the use of the bottle or bottle?
a). Always
b). Sometimes
c). Never

6. Hygiene in the home

6.1. Water quality: 

a). Not boiled
b). Chlorinated ----- c). Boiled-----

6.2. Liquid residuals:

They keep liquid residuals inside the house
a). Usually ---- Occasionally ----- c). Never-----

6.3. Solid residuals:

a). Stores garbage in tanks without a lid

b) There is garbage around and patios

c) Stores garbage in tanks with lid

Qualification for items 5 and 6 . In all cases 1 point for subsection a, 2 points for subsection $b$ and 3 points for subsection c.

Total 45 points: up to 14 points deficient, 15 - 30 average and from 31 onwards adequate 